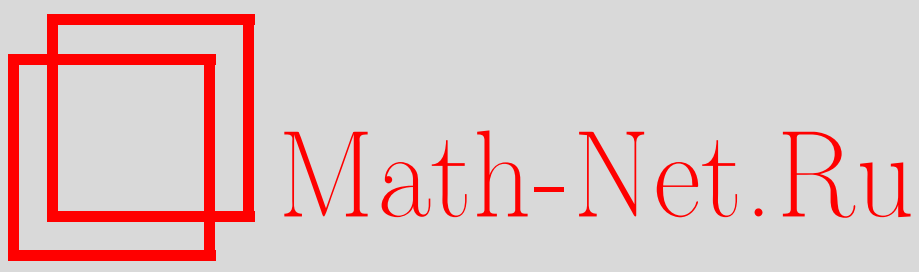

B. G. Grebenshchikov, A method of constructing almost periodic solutions to a system of neutral type with linear delay, Sibirsk. Mat. Zh., 2019, Volume 60, Number 6, 12601270

DOI: https://doi.org/10.33048/smzh.2019.60.606

Use of the all-Russian mathematical portal Math-Net.Ru implies that you have read and agreed to these terms of use http://www . mathnet.ru/eng/agreement

Download details:

IP: 18.234 .197 .8

April 26, 2023, 15:22:00

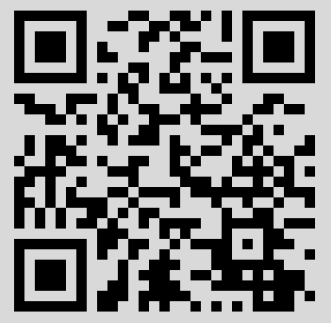


Сибирский математический журнал

Ноябрь-декабрь, 2019. Том 60, № 6

УДК $519.17+512.54$

\title{
ОДИН МЕТОД ПОСТРОЕНИЯ ПОЧТИ ПЕРИОДИЧЕСКИХ РЕШЕНИЙ ДЛЯ СИСТЕМЫ НЕЙТРАЛЬНОГО ТИПА \\ С ЛИНЕЙНЫМ ЗАПАЗДЫВАНИЕМ
}

Б. Г. Гребенщиков

\begin{abstract}
Аннотация. Изучена возможность построения почти периодического решения для одной неоднородной системы линейных дифференциальных уравнений с запаздыванием, являющимся линейной функцией аргумента (времени) при некоторых предположениях относительно правой части данной системы. Доказано, что это решение асимптотически устойчиво. Исследуется также проблема существования почти периодического решения у одной системы без нейтральных членов, при этом данное решение устойчиво.
\end{abstract}

DOI 10.33048/smzh.2019.60.606

Ключевые слова: запаздывание, почти периодические решения, устойчивость.

1. Основные определения. Рассматриваем линейное нормированное пространство $\mathbb{R}^{m}$ с нормой $\|w\|=\max _{j}\left\{w_{j}^{\top}\right\}$ (здесь $w_{j}(j=1,1, \ldots, m)$ - компоненты вектора $w, \top-$ значок транспонирования).

Норму матрицы $D=\left\{d_{i j}\right\}(i, j=1, \ldots, m)$ определим в соответствии с нормой вектора [1], а именно

$$
\|D\|=\max _{i} \sum_{j}\left|d_{i j}\right| .
$$

В пользу такого выбора говорит то, что норма вектор-функции на отрезке определяется почти сходным образом.

ОПРЕДЕЛЕНИЕ 1. Функция $f(t)$ называется почти периодической в смысле Бора, если для любого $\varepsilon>0$ существует положительное число $l(\varepsilon)$ такое, что любой отрезок $[\vartheta, \vartheta+l]$ содержит по меньшей мере одно число $T$, для которого выполнено неравенство $|f(t+T)-f(t)|<\varepsilon,-\infty<t<\infty$.

ОПРедЕлЕние 2. Решение $x\left(t, \phi(s), \phi^{\prime}(s)\right)$ системы дифференциальных уравнений с линейным запаздыванием $\gamma(t)=(1-\mu) t(\mu=$ const, $0<\mu<1)$ нейтрального типа:

$$
d x(t) / d t=A x(t)+B x(\mu t)+R d x(\mu t) / d t,
$$

определенное непрерывно дифференцируемой начальной вектор-функцией $\phi(s)$ $\left(s \in\left[\mu t_{0}, t_{0}\right]\right)$, называется устойчивым, если существует постоянная величина $\widehat{C}>0$ такая, что из условия ограниченности величины $\|\phi(s)\|+\left\|\phi^{\prime}(s)\right\|$ следует неравенство $\left\|x\left(t, \phi(s), \phi^{\prime}(s)\right)\right\|<\widehat{C}\left(t>t_{0}\right)$.

(c) 2019 Гребенщиков Б. Г. 
ОПРЕДЕЛЕНИЕ 3 . Если решение $x\left(t, \phi(s), \phi^{\prime}(s)\right)$ системы (1.2) наряду с устойчивостью обладает свойством $\lim _{t \rightarrow \infty}\left\|x\left(t, \phi(s), \phi^{\prime}(s)\right)\right\|=0$, то такое решение называется асимптотически устойчивым.

2. Постановка задачи. Рассмотрим линейную неоднородную систему нейтрального типа с линейным запаздыванием

$d x(t) / d t=A x(t)+B x(\mu t)+R d x(\mu t) / d t+f(t), \quad \mu=$ const $, 0<\mu<1, \quad t \geq t_{0}>0$.

Здесь $A, B, R$ - постоянные матрицы размера $m \times m, x(t)-m$-мерная векторфункция времени (аргумента) $t ; f(t)-m$-мерная вектор-функция, непрерывная на полуоси $[0,+\infty)$.

Решение системы (2.1) определено начальной вектор-функцией $\phi(s)$, заданной на отрезке $\left[\mu t_{0}, t_{0}\right]$, имеющей (в соответствии с определением 2 ) непрерывную производную при $\mu t_{0}<s \leq t_{0}[2$, теорема 10.6]. Отметим, что системы с линейным запаздыванием встречаются в задачах механики, физики, биологии. В частности, при исследовании процесса колебаний токоприемника движущегося локомотива при взаимодействии с контактным проводом (при учете воздействия эластичной опоры) в [3] задача сводится к изучению поведения решения неоднородного дифференциального уравнения первого порядка с линейным запаздыванием. Более сложная модель, приводящая к исследованию системы четвертого порядка, предложена в [4]. Если рассматривать колебания токоприемника при удалении локомотива от опоры, то возникают, в свою очередь, системы нейтрального типа с линейным запаздыванием. Задача прохождения токоприемником эластичной опоры имеет практическое применение при изучении устойчивости колебаний движущегося токоприемника и при определении износа контактного провода при различных скоростях движения локомотива, а также при различной величине силы нажатия полоза токоприемника на контактный провод. Учет эффекта последействия важен для правильного качественного и количественного описаний данных процессов.

Полагаем, что собственные значения $\lambda$ матрицы $A$ имеют отрицательную вещественную часть, т. е. справедливо неравенство

$$
\operatorname{Re}(\lambda)<-\delta_{1}, \quad \delta_{1}=\text { const }, \quad \delta_{1}>0 .
$$

Наряду с этим считаем, что собственные значения $\rho$ матрицы $-A^{-1} B$ меньше единицы по модулю, т. е. справедлива оценка

$$
|\rho|<\delta_{2}, \quad \delta_{2}=\text { const, } \quad 0<\delta_{2}<1 .
$$

Наконец, считаем, что оценка, подобная (2.3), справедлива и для собственных значений $\nu$ матрицы $R$, т. е.

$$
|\nu|<\delta_{3}, \quad \delta_{3}=\text { const }, \quad 0<\delta_{3}<1 .
$$

Непрерывную вектор-функцию $f(t)$ считаем равномерно ограниченной, т. е. справедливо неравенство

$$
\sup _{0 \leq t<\infty}\|f(t)\| \leq K, \quad K=\text { const }, \quad K>0
$$

(в дальнейшем наложим еще некоторые ограничения на данную вектор-функцию). 


\section{3. Ограниченность решения линейной неоднородной системы.}

Теорема 1. При выполнении неравенств (2.2)-(2.5) решение системы (2.1) ограничено.

ДокАЗАтЕльство. Ввиду положительности запаздывания $\gamma(t)$ и ограниченности величины $\left\|\phi^{\prime}(s)\right\|$ решение системы $(2.1)$ существует $[5$, теорема существования и единственности]. Заменой $\tau=\ln (t)$ сведем систему (2.1) к следующей системе с постоянным запаздыванием $\sigma=-\ln (\mu)[2]:$

$$
d z(\tau) / d \tau=e^{\tau}[A z(\tau)+B z(\tau-\sigma)+\bar{f}(\tau)]+\mu R d z(\tau-\sigma) / d \tau, \quad \bar{f}(\tau)=f\left(e^{\tau}\right) .
$$

Известно [6], что при выполнении неравенств $(2.2)-(2.4)$ решение $z^{0}(\tau)$ соответствующей однородной системы

$$
d z^{0}(\tau) / d \tau=e^{\tau}\left[A z^{0}(\tau)+B z^{0}(\tau-\sigma)\right]+\mu R d z^{0}(\tau-\sigma) / d \tau
$$

удовлетворяет экспоненциальной оценке

$$
\begin{gathered}
\left\|z^{0}(\tau)\right\| \leq \bar{M} e^{-\bar{\beta}\left(\tau-\tau_{0}\right)}\left[\sup _{\tau_{0}-\sigma \leq s \leq \tau_{0}+, 1 \leq i \leq m}\left\{\left|\bar{\phi}_{i}(s)\right|,\left|\bar{\phi}_{i}^{\prime}(s)\right|\right\}\right], \\
z^{0}(s)=\bar{\phi}(s), \quad \tau_{0}-\sigma \leq s \leq \tau_{0}, \\
\bar{M}=\mathrm{const}, \quad \bar{M}>0, \quad \bar{\beta}=\mathrm{const}, \bar{\beta}>0 .
\end{gathered}
$$

Константы $\bar{M}, \bar{\beta}$ одни и те же при любых начальных значениях $\tau_{0} \geq \tau_{0}^{*}>0$.

Полагаем $z_{n+1}(\tau)=z(\tau+n \sigma), z_{0}(\tau)=\bar{\phi}(\tau-\sigma) \bar{f}_{n+1}(\tau)=\bar{f}(n \sigma+\tau), \tau_{0} \leq$ $\tau \leq \tau_{0}+\sigma$. Тогда из системы (3.1) получаем счетную систему

$$
d z_{n+1}(\tau) / d \tau=\mu^{-n} e^{\tau}\left[A z_{n+1}(\tau)+B z_{n}(\tau)+\bar{f}_{n+1}(\tau)\right]+\mu R d z_{n}(\tau) / d \tau
$$

на конечном промежутке $\left[\tau_{0}, \tau_{0}+\sigma\right]$ при граничных условиях

$$
z_{n+1}\left(\tau_{0}\right)=z_{n}\left(\tau_{0}+\sigma\right)
$$

Пусть $U_{n}(\tau, s)=\exp \left(\mu^{-n} A\left(e^{\tau}-e^{s}\right)\right), \tau_{0} \leq s \leq \tau \leq \tau_{0}+\sigma$. Представим решение неоднородной системы (3.4) в интегральном виде (виде Коши) [2]:

$$
\begin{aligned}
z_{n+1}(\tau)=U_{n}\left(\tau, \tau_{0}\right) & z_{n}\left(\tau_{0}+\sigma\right)+\int_{\tau_{0}}^{\tau} U_{n}(\tau, s) B z_{n}(s) \frac{e^{s}}{\mu^{n}} d s \\
& +\int_{\tau_{0}}^{\tau} U_{n}(\tau, s) \mu R d z_{n}(s) / d s+\int_{\tau_{0}}^{\tau} U_{n}(\tau, s) \bar{f}_{n+1}(s) \frac{e^{s}}{\mu^{n}} d s .
\end{aligned}
$$

Если ввести норму вектор-функции $z(\tau)$ на отрезке $\left[\tau_{0}, \tau_{0}+\sigma\right]$ следующим образом [5]:

$$
\|z(\tau)\|_{\sigma}=\sup _{\substack{\tau_{0} \leq \tau \leq \tau_{0}+\sigma, 1 \leq i \leq m}}\left\{\left|z_{i}(\tau)\right|,\left|z_{i}^{\prime}(\tau)\right|\right\}, \quad \tau \in\left[\tau_{0}, \tau_{0}+\sigma\right],
$$

то при такой нормировке имеем банахово пространство $C_{\left[\tau_{0}, \tau_{0}+\sigma\right]}^{1}$ непрерывных функций, имеющих первую производную. В данном пространстве определим оператор сдвига $T_{n, \tau}$ (аналогично $[7$, гл. IV]):

$$
\begin{aligned}
T_{n, \tau} w(s)=U_{n}\left(\tau, \tau_{0}\right) w\left(\tau_{0}+\sigma\right) & +\int_{\tau_{0}}^{\tau} U_{n}(\tau, s) B w(s) \frac{e^{s}}{\mu^{n}} d s \\
& +\int_{\tau_{0}}^{\tau} U_{n}(\tau, s) \mu R d w(s) / d s, w(\tau) \in C_{\left[\tau_{0}, \tau_{0}+\sigma\right]}^{1} .
\end{aligned}
$$


Поскольку произведение операторов в банаховом пространстве определено $[8$, гл. 3], решение однородной дифференциально-разностной системы $z_{n+1}^{0}(\tau)$ (соответствующей системе (3.3)) имеет вид

$$
\begin{gathered}
z_{n+1}^{0}(\tau)=T_{n, \tau} T_{n-1, s_{n-1}} T_{n-2, s_{n-2}} \ldots T_{1, s_{1}} T_{0}\left(s_{0}\right) z_{0}(s) \\
\tau_{0} \leq s \leq s_{0} \leq s_{1} \leq \cdots \leq s_{n-1} \leq \tau \leq \tau_{0}+\sigma .
\end{gathered}
$$

Отметим некоторые свойства данного линейного оператора. Ввиду (2.2) справедливо следующее неравенство [1, гл. 1]:

$$
\left\|U_{n}(\tau, s)\right\| \leq M \exp \left(\frac{-\delta_{1}}{\mu^{n}} A\left(e^{\tau}-e^{s}\right)\right), \quad M=\mathrm{const}, \quad M>1, \tau_{0} \leq s \leq \tau \leq \tau_{0}+\sigma .
$$

Из (3.7) следует, что

$$
\left\|T_{n, \tau} w(s)\right\| \leq M\left(1+\frac{\|B\|}{\delta_{1}} \sup _{s}\|w(s)\|+\mu^{n+1} \frac{\|R\|}{\delta_{1} t_{0}} \sup _{s}\left\|w^{\prime}(s)\right\|\right),
$$

т. е. данный оператор $T_{n, \tau}$ равномерно ограничен [8, гл. 3]. Более точное свойство данного оператора вытекает из оценки (3.3), а именно

$$
\left\|T_{n, \tau} \prod_{j=0}^{n-1} T_{j, s_{j}} z_{0}(s)\right\| \leq \bar{M} q^{n-1}\left\|z_{0}\right\|_{\sigma}, \quad q=\mu^{\bar{\beta}} .
$$

Очевидно, решение неоднородного уравнения (3.6) можно представить как решение операторного уравнения

$$
z_{n+1}(\tau)=T_{n, \tau} z_{n}(s)+I_{n}(\tau) \bar{f}_{n+1}(s),
$$

где $I_{n}(\tau)$ - интегральный оператор [8. гл. 3], определенный следующим образом:

$$
I_{n}(\tau) w(s)=\int_{\tau_{0}}^{\tau} U_{n}(\tau, s) w(s) \frac{e^{s}}{\mu^{n}} d s .
$$

Отметим некоторые свойства этого оператора. Как следует из (3.2), для векторфункции $F_{n}(\tau)=I_{n}(\tau) \bar{f}_{n+1}(s)$ справедлива оценка

$$
\sup _{\tau}\left\|F_{n}(\tau)\right\| \leq \frac{M K}{\delta_{1}}
$$

отсюда оператор $I_{n}(\tau)$ равномерно ограничен. Далее, для выражения $d F_{n}(\tau) / d \tau$ имеем равенство

$$
d F_{n}(\tau) d \tau=\frac{A e^{\tau}}{\mu^{n}} F_{n}(\tau)+\frac{e^{\tau}}{\mu^{n}} \bar{f}_{n}(\tau) .
$$

Из уравнений (3.8), (3.11) следует равенство

$$
\begin{aligned}
& z_{n+1}(\tau)=T_{n, \tau} T_{n-1, s_{n-1}} T_{n-2, s_{n-2}} \ldots T_{1, s_{1}} T_{0}\left(s_{0}\right) z_{0}(s) \\
& \quad+T_{n, \tau} T_{n-1, s_{n-1}} T_{n-2, s_{n-2}} \ldots T_{1, s_{1}} I_{0}\left(s_{1}\right) \bar{f}_{1}(s) \\
& +T_{n, \tau} T_{n-1, s_{n-1}} T_{n-2, s_{n-2}} \ldots T_{2, s_{2}} I_{1}\left(s_{2}\right) \bar{f}_{2}(s)+\cdots+I_{n}(\tau) f_{n+1}(s) .
\end{aligned}
$$

Ввиду того, что справедлива оценка

$$
\sup _{s_{j}}\left\|T_{j, s_{j}} I_{j-1}\left(s_{j}\right) \bar{f}_{j}(s)\right\| \leq \frac{M^{2}}{\delta_{1}}\left[1+\frac{\|B\|}{\delta_{1}}+\frac{\|R\|}{t_{0}}\left(\frac{\|A\|}{\delta_{1}}+1\right)\right] K=\bar{L} K
$$


(она получается из соотношений $(3.3),(3.10),(3.13)$ и (3.14)), имеем

$$
\sup _{\tau}\left\|T_{n, \tau} T_{n-1, s_{n-1}} T_{n-2, s_{n-2}} \ldots T_{j, s_{j}} I_{j-1}\left(s_{j}\right) \bar{f}_{j}(s)\right\| \leq \bar{M} L K q^{n-j-1} .
$$

Тогда из соотношения (3.15) вытекает неравенство

$$
\sup _{\tau}\left\|z_{n+1}(\tau)\right\| \leq \bar{M} q^{n-1}\left\|z_{0}(\tau)\right\|_{\sigma}+\overline{L M} K\left(1+q+q^{2}+\cdots+q^{n-2}\right)+\frac{M K}{\delta_{1}},
$$

откуда получаем окончательную оценку любого $n$ :

$$
\sup _{\tau}\left\|z_{n+1}(\tau)\right\| \leq q^{n-1}\left\|z_{0}(\tau)\right\|_{\sigma}+\left[\frac{\overline{L M}}{1-q}+\frac{M}{\delta_{1}}\right] K,
$$

из которой и следует утверждение теоремы 1.

4. Построение почти периодического решения. Пусть теперь $f(t) \in$ $\Pi^{(k)}$, где $\Pi^{(k)}-$ пространство почти периодических вектор-функций, имеющих производные до порядка $k[1$, дополнение]. Полагаем, что в системе (1.1) начальная вектор-функция $\phi(s)$ тождественно нулевая, $\mu t_{0} \leq s \leq t_{0}$.

Возникает вопрос о существовании почти периодического решения системы (1.1) и его вычислении.

Теорема 2. При выполнении неравенств (2.2)-(2.4) и условий, налагаемых на вектор-функцию $f(t)$, система (1.1) имеет при любых $0<\mu<1$ почти периодическое решение $\bar{x}(t)$, которое может быть найдено методом последовательных приближений. Данное решение единственно и асимптотически устойчиво.

ДокАЗАТЕЛЬСтво. Будем искать почти периодическое решение $\bar{x}(t)$ методом последовательных приближений, полагая

$$
d x_{n+1}(t) / d t=A x_{n+1}(t)+B x_{n}(\mu t)+R d x_{n}(\mu t) / d t+f(t), \quad x_{0}=0, n=1,2 \ldots
$$

Очевидно, $x_{1}(t)=\bar{y}(t)$, где $\bar{y}(t)-$ почти периодическое решение неоднородной системы без запаздывающих членов

$$
d y(t) / d t=A y(t)+f(t) .
$$

Известно [1, дополнение], что данное (частное) решение системы (4.2) имеет вид

$$
\bar{y}(t)=\int_{-\infty}^{t} e^{A(t-s)} f(s) d s .
$$

Но производная $x_{1}^{\prime}(t)=d x_{1}(t) / d t$ является почти периодической вектор-функцией и $x_{1}^{\prime}(\mu t)$ также является почти периодической вектор-функцией $[1$, дополнение]. Последовательно интегрируя соотношения (4.1), найдем $x_{2}(t)$, и т. д.

Для доказательства сходимости последовательных приближений введем следующую вектор-функцию: $\Delta_{n+1}(t)=x_{n+1}(t)-x_{n}(t)$. Очевидно, $\Delta_{n+1}(t)$ удовлетворяют дифференциально-разностным соотношениям

$$
d \Delta_{n+1}(t) / d t=A \Delta_{n+1}(t)+B \Delta_{n}(\mu t)+R d \Delta_{n}(\mu t) / d t, \quad \Delta_{1}(t)=\bar{y}(t) .
$$

Отметим, что

$$
d x(\theta(t)) / d t=(d x(\theta(t)) / d \theta)(d \theta(t) / d t),
$$

тогда $d \Delta_{n}(\mu t) / d t=\mu d \Delta_{n}(\theta) / d \theta$. 
Последовательно получаем из (4.4) следующие равенства:

$$
\begin{gathered}
d \Delta_{1}(t) / d t=A \Delta_{1}(t)+f(t)=A \bar{y}(t)+f(t), \\
d \Delta_{2}(t) / d t=A \Delta_{2}(t)+B \Delta_{1}(\mu t)+R\left[A \Delta_{1}(\mu t)+f(\mu t)\right] \\
d \Delta_{3}(t) / d t=A \Delta_{3}(t)+B \Delta_{2}(\mu t)+R\left[A \Delta_{2}(\mu t)+B \Delta_{1}\left(\mu^{2} t\right)\right] \\
+R^{2}\left[A \Delta_{1}\left(\mu^{2} t\right)+f\left(\mu^{2} t\right)\right], \ldots \\
d \Delta_{n}(t) / d t=A \Delta_{n}(t)+B \Delta_{n-1}(\mu t)+R\left[A \Delta_{n-1}(\mu t)+B \Delta_{n-2}\left(\mu^{2} t\right)\right] \\
+R^{2}\left[A \Delta_{n-2}\left(\mu^{2} t\right)+B \Delta_{n-3}\left(\mu^{3} t\right)\right]+\cdots+R^{n-1}\left[A \Delta_{1}\left(\mu^{n-1} t\right)+f\left(\mu^{n-1} t\right)\right]
\end{gathered}
$$

(здесь $R^{2}=R \cdot R, R^{n}=R \cdot R^{n-1}$; правые части соотношений (4.5) уже не содержат «нейтральных» членов).

Ввиду того, что $f(t) \in \Pi^{(k+1)}$, ее производные также являются почти периодическими вектор-функциями. Тогда (следуя [9]), дифференцируя обе части последнего соотношения в (4.5) $j$ раз $(1 \leq j \leq k)$, получаем

$$
\begin{aligned}
& \Delta_{n}^{(j+1)}(t)=A \Delta_{n}^{(j)}(t)+\mu^{j} B \Delta_{n-1}^{(j)}(\mu t)+\mu^{j} R A \Delta_{n-1}^{(j)}(\mu t) \\
& \quad+\mu^{2 j} R B \Delta_{n-2}^{(j)}\left(\mu^{2} t\right)+\mu^{2 j} R^{2} A \Delta_{n-2}^{(j)}\left(\mu^{2} t\right)+\ldots \\
& +\mu^{(n-1)(j)} R^{n-1}\left[A \bar{y}^{(j)}\left(\mu^{n-1} t\right)+f^{(j)}\left(\mu^{n-1} t\right)\right]=A \Delta_{n}^{(j)}(t)+f_{n, k}(t) .
\end{aligned}
$$

Отметим следующие факты. В правой части соотношения (4.6) члены, содержащие линейные запаздывания, являются почти периодическими векторфункциями. Следовательно, вектор-функция $f_{n, k}(t)$ почти периодическая. Ввиду неравенства (2.2) справедлива оценка, аналогичная (3.9):

$$
\left\|e^{A(t-s)}\right\| \leq M e^{-\delta_{1}(t-s)}, \quad t_{0} \leq s<t .
$$

Наконец, вследствие (2.4) имеем неравенство [7, гл. і]

$$
\left\|R^{j}\right\| \leq M_{1}\left(\delta_{3}\right)^{j}, \quad M_{1}=\text { const }, M_{1}>1 .
$$

Пусть в (4.6) $j=k$. Рассмотрим систему (4.6). Считаем, что члены, содержащие $R^{i}, i=1,2, \ldots$, являются «возмущениями». Полагая, что «порожденное» (вектор-функцией $\left.f_{n, k}(t)\right)$ почти периодическое решение соответствующей «невозмущенной» системы имеет вид, аналогичный (4.3), и учитывая оценки (4.7) и (4.8), получаем неравенство

$$
\begin{aligned}
& \sup _{t}\left\|\Delta_{n}^{(k)}(t)\right\| \leq \frac{M \mu^{k}}{\delta_{1}}\|B\| \sup _{t}\left\|\Delta_{n-1}^{(k)}(t)\right\|+\frac{M M_{1}}{\delta_{1}}\|A\| \delta_{3} \mu^{k} \sup _{t}\left\|\Delta_{n-1}^{(k)}(\mu t)\right\| \\
&+\frac{M M_{1}}{\delta_{1}}\|B\| \delta_{3} \mu^{2 k} \sup _{t}\left\|\Delta_{n-2}^{(k)}\left(\mu^{2} t\right)\right\|+\frac{M M_{1}}{\delta_{1}}\|A\|\left(\delta_{3}\right)^{2} \mu^{2 k} \sup _{t}\left\|\Delta_{n-2}^{(k)}\left(\mu^{2} t\right)\right\|+\ldots \\
&+\frac{M M_{1}}{\delta_{1}}\left(\delta_{3}\right)^{n-1} \mu^{(n-1) k}\left[\sup _{t}\|A\|\left\|\Delta_{1}^{(k)}\left(\mu^{n-1} t\right)\right\|+\left\|f^{(k)}\left(\mu^{n-1} t\right)\right\|\right] .
\end{aligned}
$$

Полагаем

$$
u_{j}=\mu^{k j}\left(\delta_{3}\right)^{j} \sup _{t}\left\|\Delta_{j}^{(k)}\left(\mu^{j} t\right)\right\|,
$$

тогда из последнего неравенства получаем весьма грубую оценку

$$
u_{n}<\hat{b}\left[u_{n-1}+u_{n-2}+\cdots+u_{2}+u_{1}\right]+\hat{a} \bar{f}_{k} .
$$


Здесь

$$
\hat{b}=\frac{M M_{1}}{\delta_{1} \delta_{3}}\|B\|+\frac{M M_{1}}{\delta_{1}}\|A\|, \quad \hat{a}=\frac{M M_{1}}{\delta_{1}}, \quad \bar{f}_{k}=\sup _{t}\left\|f^{(k)}(t)\right\| .
$$

Если теперь обозначить $v_{n+1}=\hat{b}\left[u_{n-1}+u_{n-2}+\cdots+u_{1}\right]+\hat{a} \bar{f}_{k}$, то получим следующую цепь неравенств:

$$
v_{n+1}-v_{n}=\hat{b} u_{n} \leq \hat{b} v_{n}, \quad n=1,2 \ldots,
$$

откуда

$$
v_{n+1} \leq(1+\hat{b}) v_{n} \leq(1+\hat{b})^{2} v_{n-1} \leq \cdots \leq(1+\hat{b})^{n-1} v_{2}=(1+\hat{b})^{n-1}\left(\hat{b} u_{1}+\hat{a} u_{1}^{\prime}\right) .
$$

Но тогда

$$
u_{n} \leq(1+\hat{b})^{n-1}\left(\hat{b} u_{1}+\hat{a} \bar{f}_{k}\right)
$$

или, возвращаясь к прежним переменным $\left(\sup _{t}\left\|\Delta_{j}^{(k)}\left(\mu^{j} t\right)\right\|\right)$, имеем следующую оценку:

$$
\begin{gathered}
\sup _{t}\left\|\Delta_{n}^{(k)}(t)\right\| \leq L_{k}\left(q_{k}\right)^{n}\left[\sup _{t}\left\|\bar{y}^{(k)}(t)\right\|+\sup _{t}\left\|f^{(k)}(t)\right\|\right] \\
L_{k}=\mathrm{const}, \quad L_{k}>1, \quad q_{k}=\mu^{k} \delta_{3}(1+\hat{b}) .
\end{gathered}
$$

Полагаем натуральное число $k$ в (4.9) настолько большим, что справедливо неравенство

$$
q_{k}<1
$$

Тогда из (4.9) имеем предельное равенство

$$
\lim _{n \rightarrow \infty} \sup _{t}\left\|\Delta_{n}^{(k)}(t)\right\|=0
$$

Докажем, что такое предельное равенство справедливо и для $\sup _{t}\left\|\Delta_{n}^{(j)}(t)\right\|, j=$ $0,1, \ldots, k-1$.

Очевидно,

$$
\Delta_{n+1}^{(k-1)}(t)=-\mu^{k-1} A^{-1} B \Delta_{n}^{(k-1)}(\mu t)+A^{-1} \Delta_{n+1}^{(k)}(t)-\mu^{k-1} A^{-1} R \Delta_{n+1}^{(k)}(\mu t)
$$

(данное уравнение получается из исходного (4.6), если разрешить его относительно переменной $\left.\Delta_{n+1}^{(k-1)}(t)\right)$. Покажем (считая неоднородностью члены, содержащие величины $\left.\Delta_{n+1}^{(k)}(t), \Delta_{n+1}^{(k)}(\mu t)\right)$, что решение неоднородной разностной системы (4.10) может быть представлено в следующем виде (аналог формулы вариации постоянных $[7$, гл. i]):

$$
\begin{aligned}
& \Delta_{n}^{(k-1)}(t)=\left[-\mu^{k-1} A^{-1} B\right]^{n} \bar{y}^{(k-1)}\left(\mu^{n} t\right) \\
& +\sum_{i=0}^{n-1}\left[-\mu^{k-1} A^{-1} B\right]^{n-1-i}\left[A^{-1} \Delta_{i+2}^{(k)}\left(\mu^{n-1-i} t\right)-\mu^{k-1} A^{-1} R \Delta_{i+1}^{(k)}\left(\mu^{n-i} t\right)\right] .
\end{aligned}
$$

В самом деле, из (4.10) последовательно имеем

$$
\begin{gathered}
\Delta_{2}^{(k-1)}(t)=-\mu^{k-1} A^{-1} B \bar{y}^{(k-1)}(\mu t)+A^{-1}\left[\Delta_{2}^{(k)}(t)-\mu^{k-1} R \Delta_{1}^{(k)}(\mu t)\right] \\
\Delta_{3}^{(k-1)}(t)=-\mu^{k-1} A^{-1} B \Delta_{2}^{(k-1)}(\mu t)+A^{-1}\left[\Delta_{3}^{(k)}(t)-\mu^{k-1} R \Delta_{2}^{(k)}(\mu t)\right] \\
=\left(-\mu^{k-1} A^{-1} B\right)^{2} \bar{y}^{k-1}\left(\mu^{2} t\right)-\mu^{k-1} A^{-1} B A^{-1}\left[\Delta_{2}^{(k)}(t)-\mu^{k-1} R \Delta_{1}^{(k)}(\mu t)\right]
\end{gathered}
$$




$$
+A^{-1}\left[\Delta_{3}^{(k)}(t)-\mu^{k-1} R \Delta_{2}^{(k)}(\mu t)\right] .
$$

Аналогично

$$
\begin{gathered}
\Delta_{4}^{(k-1)}(t)=\left(-\mu^{k-1} A^{-1} B\right)^{3} \bar{y}(k-1)\left(\mu^{3} t\right) \\
\quad-\mu^{k-1} A^{-1} B A^{-1}\left[\Delta_{3}^{(k)}(\mu t)-\mu^{k-1} R \Delta_{3}^{(k)}(\mu t)\right] \\
+\left(\mu^{k-1} A^{-1} B\right)^{2} A^{-1}\left[\Delta_{2}^{(k)}\left(\mu^{2} t\right)-\mu^{k-1} R \Delta_{2}^{(k)}(t)\right]+A^{-1}\left[\Delta_{4}^{(k)}(t)-\mu^{k-1} R \Delta_{4}^{(k)}(\mu t)\right]
\end{gathered}
$$

и т. д.

Продолжая подобный процесс, получим для любых $\Delta_{n+1}^{(k-1)}(t)$ формулу (4.11). Рассмотрим уравнение (4.11). Ввиду оценки (2.3) справедливо неравенство

$$
\left\|\left(-A^{-1} B\right)^{i}\right\| \leq M_{2}\left(\delta_{2}\right)^{i}, \quad M_{2}=\text { const, } \quad M_{2}>1 .
$$

Учитывая (4.8) и (4.12), из (4.11) получаем

$$
\begin{aligned}
& \sup _{t}\left\|\Delta_{n}^{(k-1)}(t)\right\| \leq M_{2}\left(\mu^{k-1} \delta_{2}\right)^{n} \sup _{t}\left\|\bar{y}^{(k-1)}(t)\right\| \\
+ & M_{1} M_{2} \sum_{i=0}^{n-1}\left[\left(\mu^{k-1} \delta_{2}\right)^{n-1-i}\right]\left[\left\|A^{-1}\right\|\left(q_{k}+\|R\|\right)\left(q_{k}\right)^{i+1}\left[\sup _{t}\left\|\bar{y}^{(k)}(t)\right\|+\sup _{t}\left\|\bar{f}^{(k)}(t)\right\|\right] .\right.
\end{aligned}
$$

Без ограничения общности считаем, что $q_{k}<\sqrt{\delta_{2}}=\hat{q}$, что всегда выполнимо при достаточно большом $k$. Первый член в правой части (4.13) стремится к нулю при $n \rightarrow \infty$. Рассмотрим поведение оставшихся членов. Очевидно,

$$
\left(\mu^{k-1} \delta_{2}\right)^{n-1-i}\left(\delta_{2}\right)^{0.5(i+1)}=\left(\mu^{k-1} \hat{q}\right)^{n-1-i},
$$

тогда справедливы соотношения

$$
\begin{aligned}
& \sum_{i=0}^{n}\left(\mu^{k-1} \delta_{2}\right)^{n-1-i}\left(\delta_{2}\right)^{0.5(i+1)} \\
& =\left(\mu^{k-1}(\hat{q})^{2}\right)^{n}\left[\frac{1}{\mu^{k-1} \hat{q}}+\frac{1}{\left(\mu^{k-1} \hat{q}\right)^{2}}+\cdots+\frac{1}{\left(\mu^{k-1} \hat{q}\right)^{n}}\right] \\
& \quad=\left(\mu^{k-1}(\hat{q})^{2}\right)^{n} \frac{\left(\mu^{k-1} \hat{q}\right)^{-n-1}-1}{\left(\mu^{k-1} \hat{q}\right)^{-1}-1}<\frac{(\hat{q})^{n}}{1-\mu^{k-1} \hat{q}} .
\end{aligned}
$$

Отсюда

$$
\begin{gathered}
M_{1} M_{2} \sum_{i=0}^{n-1}\left[\left(\mu^{k-1} \delta_{2}\right)^{n-1-i}\right]\left[\left\|A^{-1}\right\|\left(q_{k}+\|R\|\right)\left(q_{k}\right)^{i+1}\left(\sup _{t}\left\|\bar{y}^{(k)}(t)\right\|+\sup _{t}\left\|\bar{y}^{(k+1)}(t)\right\|\right)\right] \\
<\frac{M_{1} M_{2}\left\|A^{-1}\right\|\left(q_{k}+\|R\|\right)(\hat{q})^{n}}{1-\mu^{k-1} \hat{q}}\left(\sup _{t}\left\|\bar{y}^{(k)}(t)\right\|+\sup _{t}\left\|f^{(k)}(t)\right\|\right) . \quad
\end{gathered}
$$

Из (4.13), (4.14) получаем более грубую оценку

$$
\begin{gathered}
\sup _{t}\left\|\Delta_{n}^{(k-1)}(t)\right\| \leq L_{k-1}\left(\sqrt{\delta_{2}}\right)^{n}\left[\sup _{t}\left\|\bar{y}^{(k-1)}(t)\right\|+\sup _{t}\left\|\bar{y}^{(k)}(t)\right\|+\sup _{t}\left\|f^{(k)}(t)\right\|\right] \\
L_{k-1}=\mathrm{const}, \quad L_{k-1}>1 .
\end{gathered}
$$

Если рассмотреть уравнение

$$
\Delta_{n+1}^{(k-2)}(t)=-\mu^{k-2} A^{-1} B \Delta_{n}^{(k-2)}(\mu t)+A^{-1} \Delta_{n+1}^{(k-1)}(t)-\mu^{k-2} A^{-1} R \Delta_{n+1}^{(k-1)}(\mu t),
$$


то, принимая во внимание только что полученную оценку (4.15), методами, аналогичными использованными нами, получим из (4.16) очередное неравенство

$$
\begin{aligned}
\sup _{t}\left\|\Delta_{n}^{(k-2)}(t)\right\| \leq L_{k-2}\left(\sqrt{\delta_{2}}\right)^{n}\left[\sup _{t}\left\|\bar{y}^{(k-2)}(t)\right\|+\sup _{t}\left\|\bar{y}^{(k-1)}(t)\right\|\right. \\
\left.+\sup _{t}\left\|\bar{y}^{(k)}(t)\right\|+\sup _{t}\left\|f^{(k)}(t)\right\|\right], \quad L_{k-2}=\mathrm{const}, L_{k-2}>1,
\end{aligned}
$$

и т. д. В итоге приходим к окончательной оценке

$$
\begin{gathered}
\sup _{t}\left\|\Delta_{n}(t)\right\| \leq L_{0}\left(\sqrt{\delta_{2}}\right)^{n}\left[\sum_{i=1}^{k} \sup _{t}\left\|\bar{y}^{(i)}(t)\right\|+\sup _{t}\left\|f^{(k)}(t)\right\| \sup _{t}+\|\bar{y}(t)\|\right] \\
L_{0}=\text { const, } L_{0}>1,
\end{gathered}
$$

откуда и вытекает сходимость последовательных приближений.

Как следует из теоремы 1 , любое решение неоднородной системы (1.1) ограничено. Известно [1, дополнение], что пределом $\hat{x}(t)$ равномерно сходящейся последовательности почти периодических вектор-функций $\left\{x_{n}(t)\right\}$ является также почти периодическая вектор-функция. Данное решение $\hat{x}(t)$ единственно. Действительно, пусть есть еще одно почти периодическое решение $\hat{x}_{1}(t)$. Разность этих решений $\bar{\Delta}(t)=\hat{x}(t)-\hat{x}_{1}(t)$ также почти периодическое решение, удовлетворяющее соответствующему однородному уравнению. Но тогда ввиду неравенств (2.2)-(2.4) для этого решения справедливо предельное равенство [6] $\lim _{t \rightarrow \infty} \bar{\Delta}(t)=0$; противоречие. Следовательно, $\bar{\Delta}(t)$ не является почти периодической вектор-функцией и единственным почти периодическим решением является построенное выше методом последовательных приближений решение $\hat{x}(t)$. Оно асимптотически устойчиво, поскольку, представив решение $x(t, \phi(t))$ в виде суммы частного почти периодического решения неоднородной системы $(1.1)$ и решения $x^{0}(t, \phi(t))$ однородной системы, сведем исследование к анализу асимптотических свойств соответствующей однородной системы [5, гл. ii].

Теорема 2 доказана.

5. Существование почти периодического решения для некоторых систем без нейтральных членов. Рассмотрим неоднородную систему без нейтральных членов:

$$
d y(t) / d t=A y(t)+B y(\mu t)+f(t), \quad \mu=\mathrm{const}, 0<\mu<1, \quad t \geq t_{0}>0 .
$$

Предполагаем, что справедлива оценка (2.2) и наряду с этим устойчива (но не асимптотически) разностная система

$$
y_{n+1}=-A^{-1} B y_{n} .
$$

Условия устойчивости разностной системы (5.2) приведены, например, в [7, гл. i].

Рассмотрим достаточные условия существования почти периодического решения у системы (5.1) в этом случае. Вначале докажем одну лемму для оценки решения соответствующей однородной системы.

Лемма. При выполнении условий, налагаемых на собственные числа $\lambda$ (оценка (2.2)), решение однородной системы

$$
d \hat{y}(t) / d t=A \hat{y}(t)+B \hat{y}(\mu t),
$$


определенное кусочно непрерывной начальной вектор-функцией $\phi(s)$, устойчиво.

ДокАЗАтЕЛЬство. Продифференцируем обе части системы (5.3). Получаем систему вида

$$
d \hat{y}^{\prime}(t) / d t=A \hat{y}^{\prime}(t)+\mu B \hat{y}^{\prime}(\mu t), \quad t>\frac{t_{0}}{\mu} ; \quad \hat{y}^{\prime}(t)=d \hat{y}(t) / d t .
$$

Известно [5, гл. ii], что при $t>\frac{t_{0}}{\mu}$ решение системы (5.4) существует. Очевидно, что собственные значения $\rho_{\mu}$ «вырожденной» матрицы $-\mu A^{-1} B$ меньше единицы по модулю. В [10] методами, несколько отличающимися от использованных нами при доказательстве оценки (4.18) (с учетом (3.3)), доказано неравенство

$$
\begin{aligned}
& \left\|\hat{y}^{\prime}(t)\right\| \leq \widehat{M}_{1}\left(\frac{t}{t_{0}}\right)^{-\beta} \sup _{\mu t_{0} \leq s \leq \mu^{-1} t_{0}}\|y(s)\|, \\
& \widehat{M}_{1}=\text { const, } \widehat{M}_{1}>1, \quad \beta=\text { const, } \beta>0 .
\end{aligned}
$$

Рассмотрим поведение решения системы (5.3), учитывая оценку (5.5).

Из равенства (5.3) имеем неоднородное разностное уравнение

$$
\hat{y}(t)=-A^{-1} B y(\mu t)+A^{-1} \hat{y}^{\prime}(t) .
$$

Считая величину $\hat{y}^{\prime}(t)$ неоднородностью, запишем решение системы $(5.6)$, используя аналог формулы вариации постоянных:

$$
\hat{y}(t)=\left(-A^{-1} B\right)^{n} \hat{y}\left(\mu^{n} t\right)-\sum_{j=0}^{n-1}\left(-A^{-1} B\right)^{j} A_{-1} y^{\prime}\left(\mu^{j} t\right), \quad t \in\left(t_{0} \mu^{1-n}, t_{0} \mu^{-n}\right] .
$$

Ввиду того, что разностная система (5.2) устойчива, для любого $n=1,2, \ldots$ справедливо неравенство [7, гл. і]

$$
\left\|\left(-A^{-1} B\right)^{n}\right\| \leq \widehat{M}, \quad \widehat{M}=\text { const }, \widehat{M}>1,
$$

тогда из (5.3), (5.7) имеем оценку

$$
\begin{array}{r}
\hat{y}_{n} \leq \widehat{M} y_{0}+\left\|A^{-1}\right\| \widehat{M} \sum_{j=0}^{n-1} y_{j}^{\prime}<\widehat{M} \widehat{M}_{1}\left[\frac{\left\|A^{-1}\right\|+\|A\|+\|B\|}{q(1-q)}\right] \sup _{t}\left\|y_{0}\right\|, \\
\hat{y}_{n}=\sup _{t \in\left(\mu^{1-n} t_{0}, \mu^{-n} t_{0}\right]}\|\hat{y}(t)\|, \quad y_{j}^{\prime}=\sup _{t \in\left(\mu^{1-j} t_{0}, \mu^{-j} t_{0}\right]}\left\|y^{\prime}(t)\right\|, \quad q=\mu^{\beta}, \quad 0<q<1 .
\end{array}
$$

Из (5.8) следует устойчивость системы (5.3). Лемма доказана.

Рассмотрим проблему существования почти периодического решения у системы (5.1). Ввиду того, что разностная система устойчива, но не асимптотически, строить почти периодическое решение методами, изложенными выше, не представляется возможным. Однако существует почти периодическое решение $\bar{y}^{\prime}(t)$ системы (5.4).

Теорема 3. При ограниченности величины $\left\|\int_{t_{0}}^{t} \bar{y}^{\prime}(s) d s\right\|$ система (5.1) допускает почти периодическое решение $\bar{y}(t)$.

ДокАЗАтЕльство. Поскольку собственные числа $\lambda$ матрицы $A$ удовлетворяют оценке $(2.2)$, а собственные числа $\rho_{\mu}$ матрицы $-\mu A^{-1} B-$ оценке $(2.3)$, 
строим почти периодическое решение системы $(5.4) \bar{y}^{\prime}(t)$. Ввиду того, что $\left\|\int_{t_{0}}^{t} \bar{y}^{\prime}(s) d s\right\|$ ограничен, величина $\int_{t_{0}}^{t} \bar{y}^{\prime}(s) d s$ является почти периодической вектор-функцией $[1$, дополнение], следовательно, система (5.1) допускает почти периодическое решение, которое является устойчивым, но не асимптотически.

Теорема доказана.

Следует заметить, что при этом данное почти периодическое решение может оказаться и не единственным. В самом деле, пусть $B=-A$. Тогда любое выражение $\bar{y}(t)+\bar{h}(\bar{h}-$ постоянный вектор размерности $m)$ является почти периодическим решением системы (5.8).

Отметим, что автор продолжает исследования систем, начатые ранее в $[6,11,12]$.

\section{ЛИТЕРАТУРА}

1. Демидович Б. П. Лекции по математической теории устойчивости. М.: Наука, 1967.

2. Беллман Р., Кук К. Дифференциально-разностные уравнения. М.: Мир, 1967.

3. Fox L., Mayers D. F., Ockendon J. R., Taylor A. B. On functional differential equation // Inst. Math. Appl. 1972. V. 8. P. 271-307.

4. Ockendon J., Tayler A. The dynamics of a current collection system for an electric locomotive // Proc. Soc. London. Ser. A. 1971. V. 322. P. 447-468.

5. Эльсгольц Л. Э., Норкин С. Б. Введение в теорию дифференциальных уравнений с отклоняющимся аргументом. М.: Наука, 1971.

6. Гребенщиков Б. Г. Об устойчивости стационарных систем линейных дифференциальных уравнений нейтрального типа с запаздыванием, линейно зависящим от времени // Изв. вузов. Математика. 1991. № 7. С. 69-71.

7. Халанай А., Векслер Д. Качественная теория импульсных систем. М.: Мир, 1971.

8. Барбашин Е. А. Введение в теорию устойчивости. М.: Наука, 1967.

9. Kato T., McLeod J. B. The functional differential equation $y^{\prime}(x)=a y(\lambda x)+b y(x)$ and generalisation // Lect. Notes Math. 1972. V. 280. P. 308-313.

10. Гребенщиков Б. Г., Рожков В. И. Асимптотическое поведение решения одной стационарной системы с запаздыванием // Дифференц. уравнения. 1993. Т. 29, № 5. С. 751-758.

11. Гребенщиков Б. Г. Построение почти периодических решений для одной системы с линейным запаздыванием // Сиб. мат. журн. 2011. Т. 52, № 5. С. 786-795.

12. Гребенщиков Б. Г., Рожков В. И. Почти периодические решения одной квазилинейной системы с линейным запаздыванием // Сиб. мат. журн. 1994. Т. 35, № 4. С. 768-773.

Поступила в редакцию 4 июля 2018 г.

После доработки 30 августа 2018 г.

Принята к публикации 19 декабря 2018 г.

Гребенщиков Борис Георгиевич

Уральский федеральный университет,

кафедра прикладной математики энергетического института,

ул. Мира, 19, Екатеринбург 620002

b.g.grebenshchikov@urfu.ru 